\title{
Post-Minkowskian Hamiltonians in general relativity
}

\author{
Andrea Cristofoli, ${ }^{1, *}$ N. E. J. Bjerrum-Bohr, ${ }^{1, \dagger}$ Poul H. Damgaard, ${ }^{1, \sharp}$ and Pierre Vanhove $\odot^{2,3, \S}$ \\ ${ }^{1}$ Niels Bohr International Academy and Discovery Center Niels Bohr Insitute, \\ University of Copenhagen, DK-2100 Copenhagen, Denmark \\ ${ }^{2}$ Institut de Physique Théorique, Université Paris-Saclay CEA, CNRS, \\ F-91191 Gif-sur-Yvette Cedex, France \\ ${ }^{3}$ National Research University Higher School of Economics, Russian Federation, Moscow 101000, Russia
}

(Received 9 June 2019; published 18 October 2019)

\begin{abstract}
We describe the computation of post-Minkowskian Hamiltonians in general relativity from scattering amplitudes. Using a relativistic Lippmann-Schwinger equation, we relate perturbative amplitudes of massive scalars coupled to gravity to the post-Minkowskian Hamiltonians of classical general relativity to any order in Newton's constant. We illustrate this by deriving a Hamiltonian for binary black holes without spin up to second order in the post-Minkowskian expansion and explicitly demonstrate the equivalence with the recently proposed method based on an effective field theory matching.
\end{abstract}

DOI: 10.1103/PhysRevD.100.084040

\section{INTRODUCTION}

The detection of gravitational waves by the LIGO/Virgo Collaboration has opened up the exciting possibility of testing Einstein's theory of general relativity at a new and unprecedented level, including the regime of strong gravity as probed by black holes just prior to merging. A combination of numerical relativity and analytical methods is needed in order to push theory to the level where it can provide best-fit templates from which physical parameters can be extracted. This has spurred interest in new and innovative ideas that can facilitate computations of the required twobody interaction Hamiltonians to high accuracy.

Conventionally, the calculations of effective interaction Hamiltonians have been carried out in the systematic postNewtonian expansion of general relativity. The problem can, however, be attacked from an entirely different angle, that of relativistic scattering amplitudes as computed by standard quantum field theory methods in a quantum field theory of gravity coupled to matter [1]. Modern methods of amplitude computations greatly facilitate this program [2-9]. Incoming and outgoing particles in the scattering process are taken to past and future infinity where the metric by definition is flat Minkowskian, and the full metric is

\footnotetext{
*a.cristofoli@nbi.ku.dk

†bjbohr@nbi.dk

*phdamg@nbi.ku.dk

spierre.vanhove@ipht.fr
}

Published by the American Physical Society under the terms of the Creative Commons Attribution 4.0 International license. Further distribution of this work must maintain attribution to the author(s) and the published article's title, journal citation, and DOI. Funded by SCOAP. treated perturbatively around that Minkowskian background. The classical piece of the scattering amplitude solves the scattering problem of two black holes to the given order in Newton's constant $G_{N}$. When expanding to the appropriate post-Newtonian order and defining the interaction potential with the inclusion of the required lower-order Born subtractions as explained in detail in the next section, the amplitude also contains all the information of the boundstate problem of two massive objects to the given order in the expansion in Newton's constant. For the bound-state regime, one has, on account of the virial theorem, a double expansion in both Newton's constant and momentum. However, a more daring angle of attack is to treat the bound-state problem as not expanded in momentum while still expanding to fixed order in Newton's constant. Such an approach has recently been proposed by Cheung et al. [8], and it has already been pushed one order higher in the expansion in Newton's constant [9] (and compared to the post-Newtonian expansions in Ref. [10]). Here, the method of effective field theory is used to extract the interaction Hamiltonian; the underlying Einstein-Hilbert action coupled to matter produces the classical part of the scattering amplitude, while an effective theory of two massive objects defines the interaction Hamiltonian. The correct matching between the two theories is performed by insisting that the two theories produce the same scattering amplitude to the given order in Newton's constant.

The post-Newtonian expansion (see, e.g., Refs. [11-13] for recent comprehensive reviews) of general relativity dates back to the founding days of the theory. Its perturbation theory is ideal for the low-velocity situations of planetary orbits, satellites, and large-distance effects of general relativity that occur at velocities far below the speed of light. 
In contrast to this, the computation of observables in general relativity based on relativistic scattering amplitudes is valid for all velocities, and in particular, this is the proper framework for high-energy scattering in which kinetic energies can exceed potential energies by arbitrarily large amounts. This leads naturally to what has become known in the theory of general relativity as the post-Minkowskian expansion [14-20].

Extracting the interaction energy from the relativistic scattering amplitude for consistency with the virial theorem in the bound-state problem, one would perform a double expansion where velocity $v$ and $G_{N}$ are both kept to the appropriate order. To any given order in $G_{N}$, this would imply a truncation of a Taylor-expanded amplitude in powers of momenta. There is no general argument for whether keeping higher powers of only one of the expansion parameters in the regime in which they are of comparable magnitude will increase the accuracy. Considering its potential impact, it is nevertheless of much interest to explore the consequences of keeping higherorder terms of momenta even in the bound-state regime where they would not ordinarily have been included [8-10]. We will here show how that post-Minkowskian Hamiltonian also follows directly from the full relativistic amplitude without having to perform the amplitude matching to the effective field theory, thereby explicitly showing equivalence between the two approaches $[7,8]$.

\section{PERTURBATIVE GRAVITY AS A FIELD THEORY}

We start by introducing the Einstein-Hilbert action minimally coupled to massive scalar fields $\phi_{a}$

$\mathcal{S}=\int d^{4} x \sqrt{-g}\left[\frac{R}{16 \pi G_{N}}+\frac{1}{2} \sum_{a}\left(g^{\mu \nu} \partial_{\mu} \phi_{a} \partial_{\nu} \phi_{a}-m_{a}^{2} \phi_{a}^{2}\right)\right]$,

where $R$ defines the Ricci scalar and $g \equiv \operatorname{det}\left(g_{\mu \nu}\right)$. Perturbatively, we expand the metric around a Minkowski background: $g_{\mu \nu}(x)=\eta_{\mu \nu}+\sqrt{32 \pi G_{N}} h_{\mu \nu}(x)$. At large distances, we can treat the scattering of two massive objects $m_{a}$ and $m_{b}$ as that of two pointlike particles with the same masses. This has all been well elucidated in the literature (see, e.g., Refs. [21,22]), although most focus until now seems to have been on considering the quantum mechanical effects. The way classical terms appear from the quantum mechanical loop expansion is subtle [1,23]; see Ref. [24] for a very nice and clear discussion of this issue. Instead of expanding the action (1) in terms of ordinary Feynman rules, it pays to use modern amplitude methods to extract the needed nonanalytic pieces in momentum transfer $\vec{q}$ through the appropriate cuts at loop level [2-4].

The scattering $m_{a}+m_{b} \rightarrow m_{a}+m_{b}$ mediated by gravitons at an arbitrary loop order is described by

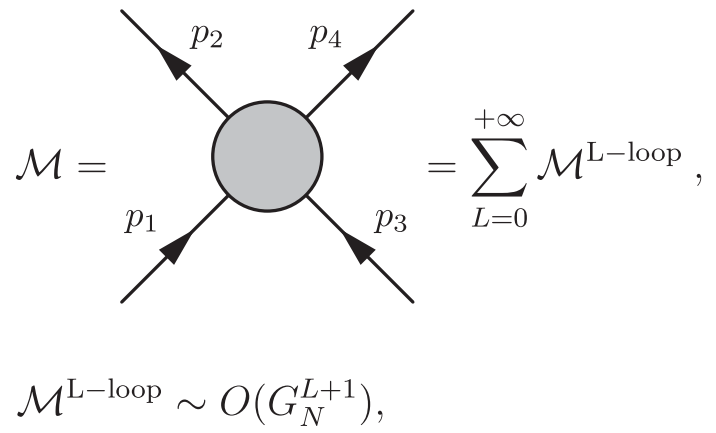

We choose the center-of-mass (c.m.) frame and parametrize the momenta as

$$
\begin{aligned}
& p_{1}^{\mu}=\left(E_{a}, \vec{p}\right), \quad p_{2}^{\mu}=\left(E_{a}, \vec{p}^{\prime}\right), \\
& p_{3}^{\mu}=\left(E_{b},-\vec{p}\right), \quad p_{4}^{\mu}=\left(E_{b},-\vec{p}^{\prime}\right),
\end{aligned}
$$

and $|\vec{p}|=\left|\vec{p}^{\prime}\right|$. We also define

$$
q^{\mu}=p_{1}^{\mu}-p_{2}^{\mu}=p_{4}^{\mu}-p_{3}^{\mu} \equiv(0,-\vec{q}), \quad \vec{q} \equiv \vec{p}^{\prime}-\vec{p},
$$

and the total energy $E_{p}=E_{a}+E_{b}$.

\section{LIPPMANN-SCHWINGER EQUATION}

It is a classical problem in perturbative scattering theory to relate the scattering amplitude $\mathcal{M}$ to an interaction potential $V$. This is typically phrased in terms of nonrelativistic quantum mechanics, but it is readily generalized to the relativistic case. Crucial in this respect is the fact that we shall consider particle solutions to the relativistic equations only. There will thus be, in the language of old-fashioned (time-ordered) perturbation theory, no back-tracking diagrams corresponding to multiparticle intermediate states. This is trivially so since we neither wish to treat the macroscopic classical objects such as heavy neutron stars as indistinguishable particles with their corresponding antiparticles nor wish to probe the scattering process in any potential annihilation channel. The classical objects that scatter will always be restricted to classical distance scales.

We now briefly outline a systematic procedure for connecting the scattering amplitude in perturbative gravity with post-Minkowskian potentials in classical general relativity. We start by introducing a bit of notation. First, we assume the existence of a relativistic one-particle Hamiltonian of only particle states describing what in bound-state problems is known as the Salpeter equation,

$\hat{\mathcal{H}}=\hat{\mathcal{H}}_{0}+\hat{V}, \quad \hat{\mathcal{H}}_{0}=\sqrt{\hat{k}^{2}+m_{a}^{2}}+\sqrt{\hat{k}^{2}+m_{b}^{2}}$,

where $\hat{V}$ is a so far unspecified potential describing our post-Minkowskian system. We also define, on a proper subset of the complex plane, the following $\mathbb{C}$-valued operators: 


$$
\begin{gathered}
\hat{G}_{0}(z) \equiv\left(z-\hat{\mathcal{H}}_{0}\right)^{-1}, \quad \hat{G}(z) \equiv(z-\hat{\mathcal{H}})^{-1} \\
\hat{T}(z) \equiv \hat{V}+\hat{V} \hat{G}(z) \hat{V} .
\end{gathered}
$$

Here, $\hat{G}_{0}$ and $\hat{G}$ are the Green's operator for the free and interacting case, while $\hat{T}$ is the off-shell scattering matrix of which the on-shell matrix elements provide the nontrivial $S$-matrix elements. We can relate the two Green's operators by means of the following operator identity:

$A^{-1}=B^{-1}+B^{-1}(B-A) A^{-1} \Rightarrow \hat{G}=\hat{G}_{0}+\hat{G}_{0} \hat{V} \hat{G}$.

Multiplying both sides of (7) by $\hat{G}_{0}$, combined with (8), one has

$$
\begin{gathered}
\hat{G}_{0} \hat{T}=\hat{G}_{0} \hat{V}+\hat{G}_{0} \hat{V} \hat{G} \hat{V}=\hat{G} \hat{V}, \\
\hat{T}(z)=\hat{V}+\hat{V} \hat{G}_{0}(z) \hat{T}(z),
\end{gathered}
$$

which is the basis for a perturbative knowledge of $\hat{T}$ and is usually known as the Lippmann-Schwinger equation.

We now take the inner product on scattering states $|p\rangle,\left|p^{\prime}\right\rangle$,

$\left\langle p|\hat{T}(z)| p^{\prime}\right\rangle=\left\langle p|\hat{V}| p^{\prime}\right\rangle+\int \frac{d^{3} k}{(2 \pi)^{3}} \frac{\langle p|\hat{V}| k\rangle\left\langle k|\hat{T}(z)| p^{\prime}\right\rangle}{z-E_{k}}$,

and use the crucial relation

$$
\lim _{\epsilon \rightarrow 0}\left\langle p\left|\hat{T}\left(E_{p}+i \epsilon\right)\right| p^{\prime}\right\rangle=\mathcal{M}\left(p, p^{\prime}\right),
$$

which provides the link to the conventionally defined scattering amplitude $\mathcal{M}$ in quantum field theory restricted to the particle sector. Substituting (12) into (11), we have a recursive relation between the amplitude and the postMinkowskian potential

$$
\mathcal{M}\left(p, p^{\prime}\right)=\left\langle p|V| p^{\prime}\right\rangle+\int \frac{d^{3} k}{(2 \pi)^{3}} \frac{\langle p|V| k\rangle \mathcal{M}\left(k, p^{\prime}\right)}{E_{p}-E_{k}+i \epsilon},
$$

Solving this equation iteratively, we can invert it in order to arrive at a relativistic equation for the potential $V$,

$$
\left\langle p|V| p^{\prime}\right\rangle=\mathcal{M}\left(p, p^{\prime}\right)-\int \frac{d^{3} k}{(2 \pi)^{3}} \frac{\mathcal{M}(p, k) \mathcal{M}\left(k, p^{\prime}\right)}{E_{p}-E_{k}+i \epsilon}+\cdots,
$$

or, in position space,

$$
V(p, r)=\int \frac{d^{3} q}{(2 \pi)^{3}} e^{i q \cdot r} V(p, q),
$$

with

$$
V(p, q) \equiv\left\langle p|V| p^{\prime}\right\rangle .
$$

At this stage, there has not been any restriction to a nonrelativistic limit. The antiparticle sector has been eliminated by hand, as dictated by the physical scattering process. We can thus regard (15) as defining a post-Minkowskian potential.

\section{POST-MINKOWSKIAN HAMILTONIANS}

\section{A. Post-Minkowskian potential to first order}

We are now ready to use the above definition of the relativistic interaction potential to describe the postMinkowskian Hamiltonian to the trivial lowest order for two massive scalars of masses $m_{a}$ and $m_{b}$ interacting with gravity. With the nonrelativistic normalization of external states,

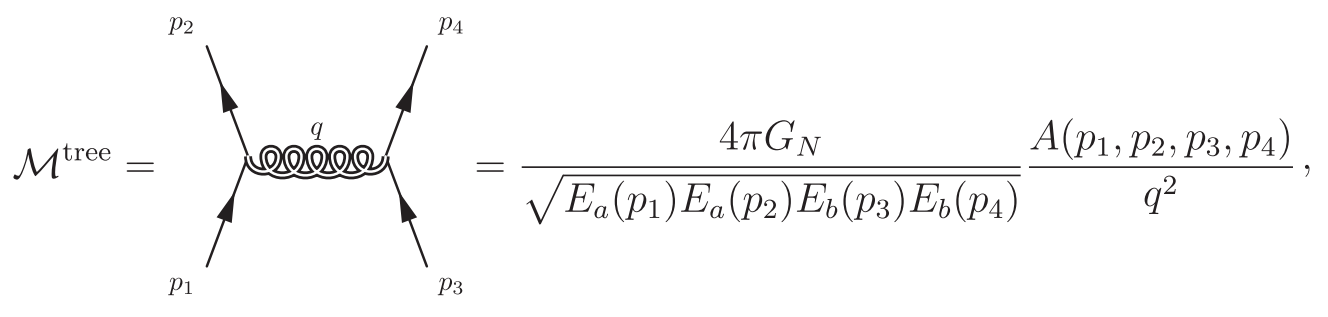

with

$A\left(p_{1}, p_{2}, p_{3}, p_{4}\right)=\left(p_{1} \cdot p_{3}\right)\left(p_{2} \cdot p_{4}\right)+\left(p_{1} \cdot p_{4}\right)\left(p_{2} \cdot p_{3}\right)-\left(p_{1} \cdot p_{2}\right)\left(p_{3} \cdot p_{4}\right)+\left(p_{1} \cdot p_{2}\right) m_{b}^{2}+\left(p_{3} \cdot p_{4}\right) m_{a}^{2}-2 m_{a}^{2} m_{b}^{2}$.

In the c.m. frame, this reduces to 
$\mathcal{M}^{\text {tree }}=-\frac{4 \pi G_{N}}{E_{a} E_{b}} \frac{\left[2\left(p_{1} \cdot p_{3}\right)^{2}-m_{a}^{2} m_{b}^{2}-|\vec{q}|^{2}\left(p_{1} \cdot p_{3}\right)\right]}{|\vec{q}|^{2}}$

with $p_{1} \cdot p_{3}=E_{a}(p) E_{b}(p)+|\vec{p}|^{2}$.

To facilitate a comparison with Ref. [8], we can write the Fourier transform as

$$
V_{1 P M}(p, r)=\frac{1}{E_{p}^{2} \xi} \frac{G_{N} c_{1}\left(p^{2}\right)}{r}+\cdots
$$

with

$$
c_{1}\left(p^{2}\right) \equiv m_{a}^{2} m_{b}^{2}-2\left(p_{1} \cdot p_{3}\right)^{2}, \quad \xi \equiv \frac{E_{a} E_{b}}{E_{p}^{2}} .
$$

The terms omitted in Eq. (20) are either ultralocal or vanishing in the classical limit. This, of course, agrees with the leading-order potential of Ref. [8], while not very easily derived in more traditional approaches.

\section{B. Post-Minkowskian potential to second order}

To consider a post-Minkowksian potential at second order in $G_{N}^{2}$, we will need to consider a contribution coming from the iterated tree-level amplitude, as dictated by (14)

$$
\begin{aligned}
V_{2 \mathrm{PM}}(p, q) & =\mathcal{M}^{1 \text {-loop }}\left(p, p^{\prime}\right)+\mathcal{M}^{\text {Iterated }}\left(p, p^{\prime}\right), \\
\mathcal{M}^{\text {Iterated }}\left(p, p^{\prime}\right) & \equiv-\int \frac{d^{d} k}{(2 \pi)^{d}} \frac{\mathcal{M}^{\text {tree }}(p, k) \mathcal{M}^{\text {tree }}\left(k, p^{\prime}\right)}{E_{p}-E_{k}+i \epsilon} .
\end{aligned}
$$

Infrared divergences are regularized by temporarily switching to $d+1$ space-time dimensions. The classical terms of the one-loop amplitude have been given elsewhere [6-8, 25-28]. They can be decomposed in terms of scalar integrals with coefficients that are independent of the exchanged 3-momentum $\vec{q}$,

$$
\begin{aligned}
\mathcal{M}^{\text {1-loop }}= & \frac{i 16 \pi^{2} G_{N}^{2}}{E_{a} E_{b}}\left(c_{\square} \mathcal{I}_{\square}+c_{\triangleright} \mathcal{I}_{\triangleright}+c_{\triangleright} \mathcal{I}_{\triangleright}\right. \\
& \left.+c_{\triangleleft} \mathcal{I}_{\triangleleft}+\cdots\right),
\end{aligned}
$$

where the symbol of each coefficient refers to the topology of the contributions involved, while the centered dots denotes quantum mechanical contributions that we neglect.

In detail, the scalar box and crossed-box integrals are given by

$$
\begin{aligned}
\mathcal{I}_{\square} & =\int \frac{d^{d+1} \ell}{(2 \pi)^{d+1}} \frac{1}{\left(\left(\ell+p_{1}\right)^{2}-m_{a}^{2}+i \varepsilon\right)\left(\left(\ell-p_{3}\right)^{2}-m_{b}^{2}+i \varepsilon\right)\left(\ell^{2}+i \varepsilon\right)\left((\ell+q)^{2}+i \varepsilon\right)}, \\
\mathcal{I}_{\bowtie} & =\int \frac{d^{d+1} \ell}{(2 \pi)^{d+1}} \frac{1}{\left(\left(\ell+p_{1}\right)^{2}-m_{a}^{2}+i \varepsilon\right)\left(\left(\ell+p_{4}\right)^{2}-m_{b}^{2}+i \varepsilon\right)\left(\ell^{2}+i \varepsilon\right)\left((\ell+q)^{2}+i \varepsilon\right)} .
\end{aligned}
$$

At leading order in the momentum transfer $\vec{q}$, the coefficients of these integrals are finite at $d=3$ and given by $[3,22]$

$$
c_{\square}=c_{\bowtie}=4\left(m_{a}^{2} m_{b}^{2}-2\left(p_{1} \cdot p_{3}\right)^{2}\right)^{2} .
$$

The scalar triangle integrals are given by

$$
\begin{aligned}
& \mathcal{I}_{\triangleright}=\int \frac{d^{d+1} \ell}{(2 \pi)^{d+1}} \frac{1}{\left((\ell+q)^{2}+i \varepsilon\right)\left(\ell^{2}+i \varepsilon\right)\left(\left(\ell+p_{1}\right)^{2}-m_{a}^{2}+i \varepsilon\right)}, \\
& \mathcal{I}_{\triangleleft}=\int \frac{d^{d+1} \ell}{(2 \pi)^{d+1}} \frac{1}{\left((\ell-q)^{2}+i \varepsilon\right)\left(\ell^{2}+i \varepsilon\right)\left(\left(\ell-p_{3}\right)^{2}-m_{b}^{2}+i \varepsilon\right)},
\end{aligned}
$$

with coefficients, at the leading order in $|\vec{q}|$ and around $d=3$, given by

$$
c_{\triangleright}=3 m_{a}^{2}\left(m_{a}^{2} m_{b}^{2}-5\left(p_{1} \cdot p_{3}\right)^{2}\right), \quad c_{\triangleleft}=3 m_{b}^{2}\left(m_{a}^{2} m_{b}^{2}-5\left(p_{1} \cdot p_{3}\right)^{2}\right) .
$$


These scalar integrals are conveniently evaluated by performing proper contour integrals in $\ell^{0}$ as explained in Ref. [7]. Doing so, we see that the box, crossed-box, and triangle contributions are given by $[22,29]$

$$
\begin{gathered}
\mathcal{I}_{\square}=-\frac{i}{16 \pi^{2}|\vec{q}|^{2}}\left(-\frac{1}{m_{a} m_{b}}+\frac{m_{a}\left(m_{a}-m_{b}\right)}{3 m_{a}^{2} m_{b}^{2}}+\frac{i \pi}{|p| E_{p}}\right) \\
\times\left(\frac{2}{3-d}-\log |\vec{q}|^{2}\right)+\cdots, \\
\mathcal{I}_{\bowtie}=-\frac{i}{16 \pi^{2}|\vec{q}|^{2}}\left(\frac{1}{m_{a} m_{b}}-\frac{m_{a}\left(m_{a}-m_{b}\right)}{3 m_{a}^{2} m_{b}^{2}}\right) \\
\times\left(\frac{2}{3-d}-\log |\vec{q}|^{2}\right)+\cdots \\
\mathcal{I}_{\triangleright}=-\frac{i}{32 m_{a}} \frac{1}{|\vec{q}|}+\cdots \\
\mathcal{I}_{\triangleleft}=-\frac{i}{32 m_{b}} \frac{1}{|\vec{q}|}+\cdots,
\end{gathered}
$$

at leading order in the $|\vec{q}|^{2}$ expansion and around $d=3$. We thus arrive at the one-loop amplitude to leading order in $|\vec{q}|^{2}$,

$$
\begin{aligned}
\mathcal{M}^{1 \text { loop }}= & \frac{\pi^{2} G_{N}^{2}}{E_{p}^{2} \xi}\left[\frac{1}{2|\vec{q}|}\left(\frac{c_{\triangleright}}{m_{a}}+\frac{c_{\triangleleft}}{m_{b}}\right)\right. \\
& \left.+\frac{i}{E_{p}} \frac{c_{\square}}{|\vec{p}|} \frac{\left(\frac{2}{3-d}-\log |\vec{q}|^{2}\right)}{\pi|\vec{q}|^{2}}\right] .
\end{aligned}
$$

The imaginary part of this which arises from the box and crossed-box integrals is the infrared-divergent Weinberg phase [30]. By restoring the $\hbar$ counting, one sees that it scales as $\hbar^{-1}$, a behavior dubbed superclassical in Ref. [24]. We will show below that it cancels in the properly defined potential, a fact already noted in the post-Newtonian expansion [25].

We next evaluate the iterated tree-level contribution given by

$$
\begin{aligned}
\mathcal{M}^{\text {Iterated }}= & -\frac{16 \pi^{2} G_{N}^{2}}{E_{a}\left(p^{2}\right) E_{b}\left(p^{2}\right)} \int \frac{d^{d} k}{(2 \pi)^{d}} \frac{A(\vec{p}, \vec{k})}{|\vec{p}-\vec{k}|^{2}} \\
& \times \frac{A\left(\vec{k}, \vec{p}^{\prime}\right)}{\left|\overrightarrow{p^{\prime}}-\vec{k}\right|^{2}} \frac{\mathcal{G}\left(p^{2}, k^{2}\right)}{E_{a}\left(k^{2}\right) E_{b}\left(k^{2}\right)},
\end{aligned}
$$

where we have introduced the Green's function

$$
\mathcal{G}\left(p^{2}, k^{2}\right)=\frac{1}{E_{p}-E_{k}+i \epsilon} .
$$

The function $A$ is the numerator of the tree-level amplitude (18) with the $k$ legs satisfying 3-momentum (but not energy) conservation. We notice that $A(\vec{p}, \vec{k})$ and $A\left(\vec{k}, \vec{p}^{\prime}\right)$ can be written as

$$
\begin{aligned}
A(\vec{p}, \vec{k}) & =\tilde{A}\left(p^{2}, k^{2}\right)+B(\vec{p}, \vec{k}), \\
A\left(\vec{k}, \vec{p}^{\prime}\right) & =\tilde{A}\left(p^{2}, k^{2}\right)+B\left(\vec{p}^{\prime}, \vec{k}\right),
\end{aligned}
$$

where $\tilde{A}$ is $\vec{q}$ independent and a function of $|\vec{p}|=p$ and $|\vec{k}|=k$. The classical contribution from the iterated Born amplitude is hence

$$
\mathcal{M}^{\text {Iterated }}=-\frac{16 \pi^{2} G_{N}^{2}}{E_{a}\left(p^{2}\right) E_{b}\left(p^{2}\right)} \int \frac{d^{d} k}{(2 \pi)^{d}} \frac{\mathcal{G}\left(p^{2}, k^{2}\right) Q\left(p^{2}, k^{2}\right)}{|\vec{p}-\vec{k}|^{2}\left|\vec{p}^{\prime}-\vec{k}\right|^{2}},
$$

where

$$
Q\left(p^{2}, k^{2}\right)=\frac{\tilde{A}^{2}\left(p^{2}, k^{2}\right)}{E_{a}\left(k^{2}\right) E_{b}\left(k^{2}\right)}
$$

We now expand $Q$ around $p^{2}$,

$$
\begin{gathered}
Q\left(p^{2}, k^{2}\right)=Q_{k=p}+\left(k^{2}-p^{2}\right) \partial_{k^{2}} Q_{k^{2}=p^{2}}+\cdots, \\
Q_{k^{2}=p^{2}}=\frac{\tilde{A}_{k^{2}=p^{2}}^{2}}{E_{a}\left(p^{2}\right) E_{b}\left(p^{2}\right)}=\frac{c_{1}^{2}}{E_{p}^{2} \xi}, \\
\partial_{k^{2}} Q_{k^{2}=p^{2}}=-\frac{1}{E_{p}^{2} \xi^{2}}\left(2 c_{1} p_{1} \cdot p_{3}+\frac{c_{1}^{2}}{2 E_{p}^{2} \xi}(1-2 \xi)\right) .
\end{gathered}
$$

The Green's function $\mathcal{G}$ likewise admits a Laurent expansion in $k^{2}$,

$$
\mathcal{G}\left(p^{2}, k^{2}\right)=\frac{2 E_{p} \xi}{p^{2}-k^{2}}+\frac{3 \xi-1}{2 E_{p} \xi}+\cdots
$$

Combining terms, the Born subtraction can hence be expressed as

$$
\begin{aligned}
\mathcal{M}^{\text {Iterated }}= & \frac{32 \pi^{2} G_{N}^{2}}{E_{p}^{3} \xi} c_{1}^{2} \int \frac{d^{d} k}{(2 \pi)^{d}} \frac{1}{|\vec{p}-\vec{k}|^{2}\left|\overrightarrow{p^{\prime}}-\vec{k}\right|^{2}\left(k^{2}-p^{2}\right)} \\
& -\frac{16 \pi^{2} G_{N}^{2}}{E_{p}^{3} \xi^{2}}\left(\frac{c_{1}^{2}(1-\xi)}{2 E_{p}^{2} \xi}+4 c_{1} p_{1} \cdot p_{3}\right) \\
& \times \int \frac{d^{d} k}{(2 \pi)^{d}} \frac{1}{|\vec{p}-\vec{k}|^{2}\left|\vec{p}^{\prime}-\vec{k}\right|^{2}}+\cdots
\end{aligned}
$$

Evaluating the remaining three-dimensional integrals, we find 


$$
\begin{aligned}
\mathcal{M}^{\text {Iterated }}= & \frac{i \pi G_{N}^{2}}{E_{p}^{3} \xi} \frac{4 c_{1}^{2}}{|\vec{p}|} \frac{\left(\log |\vec{q}|^{2}-\frac{2}{3-d}\right)}{|\vec{q}|^{2}} \\
& +\frac{2 \pi^{2} G_{N}^{2}}{E_{p}^{3} \xi^{2}|\vec{q}|}\left(\frac{c_{1}^{2}(\xi-1)}{2 E_{p}^{2} \xi}-4 c_{1} p_{1} \cdot p_{3}\right) .
\end{aligned}
$$

The second-order post-Minkowskian potential in momentum space is thus given by

$$
V_{2 \mathrm{PM}}(p, q)=\mathcal{M}^{1 \text { l-loop }}+\mathcal{M}^{\text {Iterated }}
$$

leading to

$$
\begin{aligned}
V_{2 \mathrm{PM}}(p, q)= & \frac{\pi^{2} G_{N}^{2}}{E_{p}^{2} \xi|\vec{q}|}\left[\frac{1}{2}\left(\frac{c_{\triangleright}}{m_{a}}+\frac{c_{\triangleleft}}{m_{b}}\right)\right. \\
& \left.+\frac{2}{E_{p} \xi}\left(\frac{c_{1}^{2}(\xi-1)}{2 E_{p}^{2} \xi}-4 c_{1} p_{1} \cdot p_{3}\right)\right]
\end{aligned}
$$

or, in coordinate space,

$$
\begin{aligned}
V_{2 \mathrm{PM}}(p, r)= & \frac{G_{N}^{2}}{r^{2}} \frac{1}{E_{p}^{2} \xi}\left[\frac{1}{4}\left(\frac{c_{\triangleright}}{m_{a}}+\frac{c_{\triangleleft}}{m_{b}}\right)\right. \\
& \left.+\left(\frac{c_{1}^{2}(\xi-1)}{2 E_{p}^{3} \xi^{2}}-\frac{4 c_{1} p_{1} \cdot p_{3}}{E_{p} \xi}\right)\right] .
\end{aligned}
$$

This agrees with what has been previously obtained in Ref. [8] (taking into account that $c_{1}$ here is $E^{2} \xi$ times $c_{1}$ in Ref. [8]). As expected on physical grounds, the imaginary part which is composed of superclassical and infrareddivergent pieces has canceled, leaving a finite and welldefined post-Minkowskian potential at $d=3$. That such cancellation had to occur was expected on physical grounds since the imaginary part clearly cannot affect classical motion. Interestingly, the evaluation of the same potential in $\mathcal{N}=8$ supergravity has shown no contributions coming from triangle topologies [31].

\section{Post-Minkowskian scattering angle}

In Ref. [7], a one-loop formula for the gravitational eikonal limit $[32,33]$ generalized to the scattering of two objects of different masses $m_{a}$ and $m_{b}$ was used to deduce the classical scattering angle to second post-Minkowskian order directly from the scattering amplitude. An alternative method based on the Hamiltonian [34] has recently been revived in connection with the third post-Minkowskian scattering amplitude calculation $[9,10]$, and we here briefly summarize the method at second order in $G_{N}$. Since the motion lies on a plane, we can introduce the following coordinates on the phase space $\left(r, \phi, p_{r}, p_{\phi}\right)$ so as to express the momentum in the c.m. frame as

$$
p^{2}=p_{r}^{2}+\frac{L^{2}}{r^{2}}
$$

with $L$ being the conserved angular momentum of our binary system, with constant energy $E$,

$$
\sqrt{p^{2}+m_{a}^{2}}+\sqrt{p^{2}+m_{b}^{2}}+V_{1 \mathrm{PM}}(p, r)+V_{2 \mathrm{PM}}(p, r)=E .
$$

This equation can be solved perturbatively in $G_{N}$ for $p^{2}=p^{2}(E, L, r)$ :

$$
p^{2}=p_{0}^{2}+\frac{G_{N} f_{1}}{r}+\frac{G_{N}^{2} f_{2}}{r^{2}}+\cdots .
$$

Using $s=\left(p_{1}+p_{3}\right)^{2}$,

$$
\begin{array}{ll}
p_{0}^{2} & =\frac{\left(p_{1} \cdot p_{3}\right)^{2}-m_{1}^{2} m_{2}^{2}}{s}, \quad f_{1}=-\frac{2 c_{1}}{\sqrt{s}}, \\
f_{2} & =-\frac{1}{2 \sqrt{s}}\left(\frac{c_{\triangleright}}{m_{a}}+\frac{c_{\triangleleft}}{m_{b}}\right) .
\end{array}
$$

It is straightforward to derive the expression for the change in the angular variable $\phi$ during scattering (see, for instance, Refs. [16,34]),

$$
\Delta \phi=\pi+\chi(E, L)
$$

where the scattering angle is given by

$$
\chi(E, L)=-2 \int_{r_{\min }}^{+\infty} d r \frac{\partial p_{r}}{\partial L}-\pi .
$$

Here, $r_{\min }$ is the positive root for the condition of the turning point at $p_{r}=0$ with

$$
p_{r}=\sqrt{p_{0}^{2}-\frac{L^{2}}{r^{2}}+\frac{G_{N} f_{1}}{r}+\frac{G_{N}^{2} f_{2}}{r^{2}}} .
$$

Introducing $r_{0} \equiv L / p_{0}$, we note that $p_{r}$ can be rewritten as

$$
\begin{aligned}
p_{r} & =\frac{p_{0}}{r} \sqrt{r^{2}+r \frac{G_{N} f_{1}}{p_{0}^{2}}+\frac{G_{N}^{2} f_{2}}{p_{0}^{2}}-r_{0}^{2}} \\
& =\frac{p_{0}}{r} \sqrt{r-r^{+}} \sqrt{r-r^{-}}, \\
r^{ \pm} & =-\frac{G_{N} f_{1}}{2 p_{0}^{2}} \pm \sqrt{\frac{G_{N}^{2} f_{1}^{2}}{4 p_{0}^{4}}-\frac{G_{N}^{2} f_{2}}{p_{0}^{2}}+r_{0}^{2}} .
\end{aligned}
$$

Since $r_{\min }=r^{+}$, the scattering angle becomes

$$
\chi(E, L)=2 \int_{r^{+}}^{+\infty} \frac{d r}{r} \frac{r_{0}}{\sqrt{\left(r-r^{+}\right)\left(r-r^{-}\right)}}-\pi .
$$

The integral so expressed can be performed analytically without the need of regularization. We get 


$$
\chi(E, L)=\frac{4 r_{0}}{\sqrt{-r^{+} r^{-}}} \arccos \sqrt{\frac{r^{+}}{r^{+}-r^{-}}}-\pi .
$$

Taylor expanding the scattering angle to second postMinkowskian order, we arrive at the final result

$$
\chi(E, L)=\frac{G_{N} f_{1}}{p_{0} L}+\frac{G_{N}^{2} f_{2} \pi}{2 L^{2}}+\cdots .
$$

In terms of $\hat{\mathcal{M}}^{2} \equiv s-m_{a}^{2}-m_{b}^{2}$ and the impact parameter $b$, where $L=p b$, we have

$$
\begin{aligned}
\chi(E, b)= & \frac{4 G_{N} s}{b}\left[\frac{\hat{\mathcal{M}}^{4}-2 m_{a}^{2} m_{b}^{2}}{\hat{\mathcal{M}}^{4}-4 m_{a}^{2} m_{b}^{2}}\right. \\
& \left.+\frac{3 \pi}{16} \frac{G_{N}\left(m_{a}+m_{b}\right)}{b} \frac{5 \hat{\mathcal{M}}^{4}-4 m_{a}^{2} m_{b}^{2}}{\hat{\mathcal{M}}^{4}-4 m_{a}^{2} m_{b}^{2}}\right],
\end{aligned}
$$

which agrees with the result of Ref. [14] at second postMinkowskian order. In particular, since $f_{1}$ and $f_{2}$ do not depend on box topologies (54), also the scattering angle (62) receives no contributions from these, a known fact from the eikonal approach in four dimensions. The details of the calculation based on the Hamiltonian is, on the surface, quite different from the eikonal approach. It would be interesting to establish the precise link between the two, first identifying the precise exponentiation formula for the eikonal limit beyond second post-Minkowskian order.

\section{CONCLUSION}

Using the conventional approach to determining the interaction potential in perturbative gravity, we have demonstrated that it can be extended to the relativistic setting by means of a one-particle Hamiltonian and associated Salpeter equation. We have used the Lippmann-Schwinger equation to straightforwardly derive the needed Born subtractions at arbitrary loop order. The resulting Fourier-transformed postMinkowskian Hamiltonian

$$
\begin{aligned}
\mathcal{H}_{2 \mathrm{PM}}(p, r)= & \sqrt{p^{2}+m_{a}^{2}}+\sqrt{p^{2}+m_{b}^{2}}+V_{1 \mathrm{PM}}(p, r) \\
& +V_{2 \mathrm{PM}}(p, r)
\end{aligned}
$$

agrees with the one derived in Ref. [8] based on an effective field theory expansion in operators that can contribute to the given order, supplemented with the matching condition that the scattering amplitude as computed in the effective theory agrees with the one computed from the full one-loop expression of the Einstein-Hilbert action (plus scalars).

The resulting post-Minkowskian Salpeter equation is not an effective low-energy theory (momentum is not limited) but rather a small $|\vec{q}| / m$ approximation in which small momentum is exchanged and only particle states are summed over. It is encouraging that preliminary results indicate that the corresponding two-loop Hamiltonian [9] may improve the computation of two-body dynamics as compared to the conventional post-Newtonian expansion for bound states [10]. The post-Minkowskian Hamiltonian also appears to provide a shortcut towards computing the scattering angle without first demonstrating exponentiation (and potential correction terms) as in the eikonal approach. It would be interesting to demonstrate the equivalence between those two scattering angle computations in all generality.

\section{ACKNOWLEDGMENTS}

This work has been based partly on funding from the European Union's Horizon 2020 research and innovation programme under the Marie Skłodowska-Curie Grant No. 764850 ("SAGEX"). The work of N. E. J. B.-B. and P.H.D. was supported in part by the Danish National Research Foundation (DNRF91). N. E. J. B.-B. in addition acknowledges partial support from the Carlsberg Foundation. The research of P. V. has received funding the ANR grant "Amplitudes," Grant No. ANR-17- CE310001-01, and is partially supported by Laboratory of Mirror Symmetry NRU HSE, RF Government grant, Grant No. 14.641.31.0001. P. V. also thanks the Galileo Galilei Institute for Theoretical Physics and INFN for hospitality and partial support during the workshop "String Theory from a worldsheet perspective" during which part of this work was done.
[1] Y. Iwasaki, Quantum theory of gravitation vs classical theory. Fourth-order potential, Prog. Theor. Phys. 46, 1587 (1971).

[2] D. Neill and I. Z. Rothstein, Classical space-times from the S matrix, Nucl. Phys. B877, 177 (2013).
[3] N. E. J. Bjerrum-Bohr, J. F. Donoghue, and P. Vanhove, On-shell techniques and Universal results in quantum gravity, J. High Energy Phys. 02 (2014) 111.

[4] V. Vaidya, Gravitational spin Hamiltonians from the $\mathrm{S}$ matrix, Phys. Rev. D 91, 024017 (2015). 
[5] F. Cachazo and A. Guevara, Leading singularities and classical gravitational scattering, arXiv:1705.10262.

[6] A. Guevara, Holomorphic classical limit for spin effects in gravitational and electromagnetic scattering, J. High Energy Phys. 04 (2019) 033.

[7] N. E. J. Bjerrum-Bohr, P. H. Damgaard, G. Festuccia, L. Planté, and P. Vanhove, General Relativity from Scattering Amplitudes, Phys. Rev. Lett. 121, 171601 (2018).

[8] C. Cheung, I. Z. Rothstein, and M. P. Solon, From Scattering Amplitudes to Classical Potentials in the Post-Minkowskian Expansion, Phys. Rev. Lett. 121, 251101 (2018).

[9] Z. Bern, C. Cheung, R. Roiban, C. H. Shen, M. P. Solon, and M. Zeng, Scattering Amplitudes and the Conservative Hamiltonian for Binary Systems at Third Post-Minkowskian Order, Phys. Rev. Lett. 122, 201603 (2019).

[10] A. Antonelli, A. Buonanno, J. Steinhoff, M. van de Meent, and J. Vines, Energetics of two-body Hamiltonians in postMinkowskian gravity, Phys. Rev. D 99, 104004 (2019).

[11] L. Blanchet, Gravitational radiation from post-Newtonian sources and inspiralling compact binaries, Living Rev. Relativity 17, 2 (2014).

[12] M. Levi, Effective field theories of post-Newtonian gravity: A comprehensive review, arXiv:1807.01699.

[13] A. Cristofoli, An effective field theory approach to the twobody problem in general relativity, Master thesis, Padua University, 2018.

[14] K. Westpfahl, High-speed scattering of charged and uncharged particles in general relativity, Fortschr. Phys. 33, 417 (1985).

[15] T. Ledvinka, G. Schafer, and J. Bicak, Relativistic ClosedForm Hamiltonian for Many-Body Gravitating Systems in the Post-Minkowskian Approximation, Phys. Rev. Lett. 100, 251101 (2008).

[16] T. Damour, Gravitational scattering, post-Minkowskian approximation and effective one-body theory, Phys. Rev. D 94, 104015 (2016).

[17] D. Bini and T. Damour, Gravitational spin-orbit coupling in binary systems at the second post-Minkowskian approximation, Phys. Rev. D 98, 044036 (2018).

[18] L. Blanchet and A. S. Fokas, Equations of motion of selfgravitating $N$-body systems in the first post-Minkowskian approximation, Phys. Rev. D 98, 084005 (2018).

[19] T. Damour, High-energy gravitational scattering and the general relativistic two-body problem, Phys. Rev. D 97, 044038 (2018).
[20] A. Guevara, A. Ochirov, and J. Vines, Scattering of spinning black holes from exponentiated soft factors, J. High Energy Phys. 09 (2019) 056.

[21] J.F. Donoghue, General relativity as an effective field theory: The leading quantum corrections, Phys. Rev. D 50, 3874 (1994).

[22] N. E. J. Bjerrum-Bohr, J. F. Donoghue, and B. R. Holstein, Quantum gravitational corrections to the nonrelativistic scattering potential of two masses, Phys. Rev. D 67, 084033 (2003); Erratum, Phys. Rev. D71, 069903 (2005).

[23] B. R. Holstein and J.F. Donoghue, Classical Physics and Quantum Loops, Phys. Rev. Lett. 93, 201602 (2004).

[24] D. A. Kosower, B. Maybee, and D. O'Connell, Amplitudes, observables, and classical scattering, J. High Energy Phys. 02 (2019) 137.

[25] B. R. Holstein and A. Ross, Spin effects in long range gravitational scattering, arXiv:0802.0716; , Spin effects in long range electromagnetic scattering, arXiv:0802.0715.

[26] A. Koemans Collado, P. Di Vecchia, and R. Russo, Revisiting the 2PM eikonal and the dynamics of binary black holes, arXiv:1904.02667.

[27] A. Brandhuber and G. Travaglini, On higher-derivative effects on the gravitational potential and particle bending, arXiv:1905.05657.

[28] W. T. Emond and N. Moynihan, Scattering amplitudes, black holes and leading singularities in Cubic theories of gravity, arXiv:1905.08213.

[29] J. F. Donoghue and T. Torma, On the power counting of loop diagrams in general relativity, Phys. Rev. D 54, 4963 (1996).

[30] S. Weinberg, Infrared photons and gravitons, Phys. Rev. 140, B516 (1965).

[31] S. Caron-Huot and Z. Zahraee, Integrability of black hole orbits in maximal supergravity, J. High Energy Phys. 07 (2019) 179.

[32] D. N. Kabat and M. Ortiz, Eikonal quantum gravity and Planckian scattering, Nucl. Phys. B388, 570 (1992).

[33] R. Akhoury, R. Saotome, and G. Sterman, High energy scattering in perturbative quantum gravity at next to leading power, arXiv:1308.5204.

[34] T. Damour and G. Schaefer, Higher order relativistic Periastron advances and binary pulsars, Nuovo Cimento B 101, 127 (1988). 